\section{Somatropin-Biosimilar im Fokus}

Im Jahr 2006 wurde das weltweit erste Nachfolge-Biopharmakon (Biosimilar) des menschlichen Wachstumshormons in der EU zugelassen. Zur Zulassungsstudie des Präparats (Omnitrope ${ }^{\circledR}$ ) liegen mittlerweile die Sieben-Jahres-Daten vor. Prof. Dr. Hans Peter Schwarz aus München präsentierte die wichtigsten Ergebnisse.

In die Studie wurden 89 Kinder mit Wachstumsstörungen auf Grund eines Mangels an Wachstumshormon (Growth Hormone, $\mathrm{GH}$ ) eingeschlossen. Sie erhielten randomisiert für neun Monate eines der beiden Präparate in der Dosis von 0,1 IU/kg/Tag subkutan zur Schlafenszeit. Auch nach über 84 Monaten bestätigte sich ein vergleich- bares Sicherheitsprofil beider Medikamente mit geringgradigen Nebenwirkungen und einer nur passageren Antikörperbildung bei drei Kindern.

Die Wirkungen auf Körpergröße, Wachstumsgeschwindigkeit und Serumkonzentration des Insuline-like Growth Factor (IGF-1) waren in beiden Studienarmen ebenfalls vergleichbar. Es ergaben sich keine Probleme, wenn die Kinder von einer rekombinanten $\mathrm{GH}$-Formulierung auf die andere umgestellt wurden.

In der vorliegenden Studie, so Schwarz, wurden Wirksamkeit, Unbedenklichkeit und Verträglichkeit des Nachfolgepräparats belegt. Nach seiner Ansicht kann das Ergebnis die- ser Studie prinzipiell auch auf alle anderen für Omnitrope ${ }^{\circledR}$ zugelassenen Indikationen übertragen werden: Wachstumsstörungen bei Prader-Willi- und Ullrich-Turner-Syndrom, bei chronischer Niereninsuffizienz sowie bei kleinwüchsigen Kindern und Jugendlichen im Rahmen eines Small Gestational Age (SGA). Speziell bei der letztgenannten Indikation wird eine europäische Phase-IV-Studie die Ausbildung möglicher diabetogener Effekte der Therapie mit rekombinantem $\mathrm{GH}$ klären.

TUK

Pressekonferenz „Ein halbes Jahrhundert Pionierleistung - Höchste Ansprüche an die Qualität von Sandoz Biosimilars". Kundl/ Österreich, 6. November 2009. Veranstalter: Sandoz Pharmaceuticals, Holzkirchen

\title{
Engagement für Kindergesundheit
}

- Damit Medikamente von Kindern akzeptiert werden, sollten sie gut schmecken. Bei flüssigen Arzneimitteln ist es außerdem hilfreich, wenn Dosierhilfen wie Messbecher und spezielle Dosierspritzen zur Verfügung stehen. Verschiedene Hilfsmittel und Maßnahmen, die zu einer besseren Versorgung von Kindern beitragen, bietet die Hexal AG an, die sich auf diese Weise besonders für die Kindergesundheit engagieren will. Aufgrund der Rabattverträge wird dieses Engagement allerdings nicht mehr honoriert. Dr. Andreas Busse, Tegernsee, bedauerte, dass Kinder wegen der Rabattverträge oft das billigste Arzneimittel bekommen, obwohl es einen schlechteren Geschmack hat und von den Kindern häufiger wieder ausgespuckt wird, sodass neue Verordnungen und zusätzliche Ausgaben notwendig werden.

Ein weiterer Baustein der Initiative Kindergesundheit ist die ZAK-Datenbank, die einen Beitrag zur Arzneimittelsicherheit bei Kindern leistet. Sie enthält Informationen über mehr als 2.000 für Kinder zugelassene Arzneimittel. In der Online-Datenbank www.zak-kinderarzneimittel.de ist leicht zu recherchieren, welche Wirkstoffe für pädiatrische Erkrankungen zugelassen sind und in welchen Darreichungsformen sie zur Verfügung stehen.

Auch die neue Internetseite www.meinkind.hexal.de, die sich an Eltern richtet, ist ein Bestandteil der Initiative. Dort finden die
Eltern unter anderem allgemeine Informationen über die wichtigsten Kinderkrankheiten, die Anwendung von Arzneimitteln bei Kindern und die Zuzahlungen. Praktisch erscheint vor allem der individuelle Vorsorge- und Impfkalender. Nach Eingabe des Geburtsdatums wird eine Liste erstellt, auf der die Eltern ablesen können, wann welche Vorsorgeuntersuchungen bzw. Impfungen erforderlich sind.

Busse hob besonders das Notfallmanual hervor, denn jedes Jahr verunglückt rund eine halbe Million Kinder so schwer, dass sie ärztliche Hilfe brauchen, so Busse. Die meisten Unfälle passieren zuhause, sodass die Eltern die ersten sind, die handeln müssen. Die wichtigsten Tipps zur Ersten Hilfe bei Verbrennungen, Vergiftungen und anderen Notfällen finden die Eltern ebenfalls unter www.mein-kind.hexal.de.

jn

Pressekonferenz „Kindergesundheit braucht Partner - eine Online-Initiative der Hexal AG“. München, 16. Dezember 2009. Veranstalter: Hexal AG, Holzkirchen
Neuer Therapiebegleiter

Aktuelle Daten zeigen, dass die Therapietreue bei der spezifischen Immuntherapie (SIT) unzureichend ist. Nur ein Viertel bis ein Drittel aller Patienten führt die SIT auch im dritten Jahr noch durch. Da die mangelnde Compliance den langfristigen Therapieerfolg einschränkt, muss nach Möglichkeiten gesucht werden, die Therapietreue der Patienten zu erhöhen.

Als ein wichtiger Helfer in diesem Zusammenhang könnte sich der THErapieOrganizer für Allergiker - kurz THEO - erweisen. Dieser hochwertige Allergietherapie-Pass wurde speziell entwickelt, um die Patienten oder auch deren Eltern zur Durchführung einer dreijährigen Hyposensibilisierung zu motivieren. Dazu wird nicht nur detailgenau die gesamte Behandlung dokumentiert. Der Organizer bietet darüber hinaus auch viele Informationen.

THEO kann kostenlos direkt bei Allergopharma Joachim Ganzer KG angefordert werden per Telefon (o 40) 727 65-0, Fax (o 40) 7227713 oder auch per E-Mail an info@allergopharma.de.

Nach Informationen von Allergopharma, Reinbek 